The authors suggest that the penile-bulb dose threshold for potency lies between 40Gy and 50 Gy.

Controversy continues over whether penilebulb dose influences ED merely as a surrogate for doses to other structures. Mangar et al. observed a strong correlation between radiation doses received by the penile bulb, crura, and cavernosa. They recommend that the penile bulb should be excluded from the high-dose area when preparing conformal radiotherapy plans for men with prostate cancer.

Original article Mangar SA et al. (2006) Evaluating the relationship between erectile dysfunction and dose received by the penile bulb: using data from a randomised controlled trial of conformal radiotherapy in prostate cancer (MRC RT01, ISRCTN47772397). Radiother Oncol 80: 355-362

\section{Predicting disease progression from PSA doubling time}

The majority of diagnosed prostate cancers will never progress to the point of needing treatment. Physicians face the challenge of identifying which patients will progress and require active treatment, and which patients can be safely managed by observation alone. Zhang et al. have now proposed guidelines based on the results of their new, prospective, cohort study.

Between 1995 and 2005, 231 men with prostate cancer at low risk of progression (stage T1b-T2b NOM0 disease, Gleason score $\leq 7$, and PSA level $<15 \mathrm{ng} / \mathrm{ml}$ ) were managed with observation. Over the course of the study, 93 patients were reclassified as being at high risk of disease progression and were given appropriate active treatment; data from after reclassification was not included in the analysis. Before any active treatment was given, Zhang et al. used a computer model to predict the evolution of serial PSA levels, which were later compared with actual group and individual PSA doubling times.

Two average prediction lines were producedone for the high-risk group and one for the lowrisk group. The actual average PSA doubling time was 2.97 years in the high-risk group and 6.54 years in the low-risk group. Zhang et al. suggest that active treatment should be initiated if $>40 \%$ of the patient's measurements lie above the predicted high-risk line, and that observation can be relaxed if $>35 \%$ of the patient's measurements lie below the predicted low-risk line.

Original article Zhang L et al. (2006) Modeling prostate specific antigen kinetics in patients on active surveillance. J Urol 176: 1392-1398

\section{Vardenafil improves erectile function in men with type 1 diabetes}

Ziegler et al. have carried out a prospective, randomized study assessing the safety and efficacy of flexible-dose vardenafil therapy in men with type 1 diabetes and erectile dysfunction (ED).

In this double-blind, placebo-controlled study, 302 phosphodiesterase-5-inhibitor-naive men with type 1 diabetes and ED were randomly assigned to receive either flexible-dose vardenafil $(5-20 \mathrm{mg}, n=154)$ or placebo $(n=149)$ for 12 weeks. The primary outcome measures were patient responses to Sexual Encounter Profile questions regarding successful intercoursespecifically, those relating to vaginal insertion and maintenance of erection. Additional measures included the erectile-function-domain score of the International Index of Erectile Function after 12 weeks of vardenafil treatment or placebo.

The results showed that, at 4,8 , and 12 weeks, the rate of successful vaginal insertion was significantly increased with vardenafil compared with placebo $(P<0.0001)$. Similarly, the mean rate of maintained erections allowing successful intercourse was greater with vardenafil at all time points. Treatment with vardenafil also improved the erectile function domain score $(P<0.0001)$. Vardenafil was well tolerated over the study period. The frequency of adverse effects was low; they included flushing (2.5\%) and headaches (3.1\%).

The majority of patients in the study had moderate-to-severe ED; the results are, therefore, encouraging for the treatment of ED in men with difficult-to-treat type 1 diabetes.

Original article Ziegler D et al. (2006) Efficacy and safety of flexible-dose vardenafil in men with type 1 diabetes and erectile dysfunction. J Sex Med 3: 883-891

\section{Regular NSAID use might protect against prostate enlargement}

The results of a population-based study conducted by a group from the Mayo Clinic indicate 